\title{
How do we decide? Knowledge? Experience? Research?
}

\author{
Elizabeth Kay \\ Editor, EBD
}

$\mathrm{P}$ roponents of evidence based practice want, among other things, to speed up the implementation of innovations into practice. It is said that currently, in the UK, it takes on average 17 years for innovations with proven benefit, to be fully accepted and adopted by the practising community. This implies that for 17 years patients are not receiving the best care available.

Whilst those who read this journal are unlikely to be among them, there are those who feel that the rigour of evidence based practice undermines clinical judgement which is built on real-world experience. Indeed, the feeling that (in particular), evidence based guidelines undermine professional autonomy and identity, seem not to be uncommon among the practitioners I have spoken to.

As I see it, the difficulty with implementing research findings in practice is that well conducted research, by definition, is carried out in controlled circumstances, with the number of potential confounding factors reduced to a minimum. By contrast, the context in which most clinical decisions are made and operative procedures undertaken, are infinitely complex. Dental teams make decisions and take actions in circumstances where any number of potentially conflicting roles and duties prevail. Dental teams not only have to reach the highest possible standards in the clinical domain (diagnosing, prescribing, restoring, extracting, explaining, referring) but also in managerial and technical domains (managing personnel and patients, handling and procuring materials, quality assurance, staff training, IT management). In addition they are expected to contribute to public health (disease prevention, dental health education, local community development) whilst also maintaining their continuing professional development, reading journals, and relating to professional organisations.

It would seem then, that no research project, theory, or evidence based guideline could ever be expected to be appropriate in all the possible combinations of circumstances created by variations in all the aspects of practising life listed above. However, decision making, in whatever domain, ultimately rests on the participants' assessment of risk - at which people are notoriously bad. This is particularly true if experience is used as the guide. A dentist's assessment of the risks (and benefits) of a particular treatment strategy will be influenced in part by whether he or she has experienced the event before. For example, if every tooth with radiographic evidence of caries is investigated and found to have deep decay, that dentist will tend to have a very low threshold of suspicion regarding radiographic anomalies. He/she will tend to believe that even minimal radiographic evidence of what may appear to be very early caries, may be highly indicative of advanced disease.

Another consideration, when arguing that experience alone is sufficient to underpin decision making, is the fact that research has shown that people tend to remember surprising or distressing events more clearly than other happenings in their lives. They are therefore likely to overestimate the frequency of such events. So again, their assessment of risk is biased. For example, if three days after completing a root canal treatment, one of your patients experiences acute and severe symptoms, including swelling and pyrexia, you would find it surprising, and not a little distressing. And even if you had previously completed 99 very similar treatments with $100 \%$ success, you would be unlikely to gauge the probability of success to be $99 \%$ (which would be accurate) when another similar case presented.

Finally, a further issue to consider is that people tend to estimate probabilities in a way which enhances their own image of themselves. They will therefore be likely to overestimate their own success rates. To give an example of this, it has been shown that junior doctors in acute medical wards can quite accurately estimate the overall patient survival rates. However, when asked to estimate the survival of their own patients the estimates are inflated by a considerable amount. This trait is, somewhat unfortunately, termed 'ego bias' and sadly, we all suffer from it!

The innate biases described above, and to which we are all prone, means that in order to accurately be able to assess the probability or likelihood of a particular outcome, we must assimilate both the results of research plus systematic reviews of one's own performance (internal audit).

To put all this in context, a dentist may have learnt from reading Evidence-Based Dentistry that, for example, in one study, $50 \%$ of restorations failed within eight years of placement. But the same dentist may not accept that this rate of attrition applies to his own fillings and that his restoration survival rate would be much better. This would be likely to lead the dentist to err on the side of always filling questionably carious teeth. In contrast, a dentist who accepted that failure rates are high, and who believed that the research reflects their own restoration failure rate, would be likely to delay restoration of teeth for as long as possible as the commencement of the restorative cycle will ultimately lead to the demise of the tooth.

The way forward therefore rests on us continuing to meld research evidence with knowledge derived from experience. This can only happen if those in practice are aware of the research evidence, but that they also influence the conduct of research to ensure that it addresses the uncertainties they face each day in practice. If we create systems so that research evidence and personal knowledge can be combined and inform each other, we will help to make decision making easier and will ensure that each decision made in practice brings patient benefit.

Evidence-Based Dentistry (2020) 21, 4. https://doi.org/10.1038/s41432-020-0087-5 